\title{
Editorial
}

\section{Integrity, immunity, reactivity, restorativity: Biolessons off brain abscess}

\author{
Manu Kothari, Atul Goel ${ }^{1}$ \\ Departments of Anatomy and ${ }^{1}$ Neurosurgery, King Edward Memorial Hospital and Seth G.S. Medical College, Parel, Mumbai, India
}

\begin{abstract}
A brain abscess (BA) as an ensconced pocket of pus is no more no less than an abscess on the toe or the torso. It begs to be drained along the Hiltonean maxim: wherever there is pus, let it out. It is BA's deep and 'dangerous' location that needs diagnostic and operative wizardry comprising THEOS-SLAM-AAAA. ${ }^{[1]}$ THEOS (=religion) implies Technicized High Element Of (Diagnostic) Suspicion. SLAM - meaning winning all the tricks of the game- reads as Speedily Locate, Adroitly Manage, by AAAA- Aspiration and/orAblation, Antibiotics and Antiepileptics. What biolessons could BA offer?

The term intelligence connotes 'reading between the lines', from inter $=$ between and legere $=$ to grasp, read, choose, pick up. BA's acknowledged uncommonness, bordering on rarity illustrates the immunity that the neuraxis exhibits against patently pyogenic microbes. The evolution and nature of BA manifests brain's reasoned reactivity, or mindful munity. The alacrity with which BA responds to timely measures exhibits the animal body's restitutivity or restorativity. And encompassing these three bioforces and a lot more is integrity. It is time that these bioforces comprising Vis Medicatrix Naturae - the Healing Power of Nature- were brought into a broader relief.
\end{abstract}

Macfarlane Burnet got his Nobel in 1960 for concepts that spawned the idea of body-cells as 'self' rejecting anything perceived as non-self. In 1962, Burnet published a mini-classic Integrity of the Body, wherein he evolved the idea that the chief function of the animal immune system is to keep intact the body's integrity or wholeness by rejecting whatever that is alien to it.

In Cellular Immunology Burnet interestingly observes: "Primitive neurons seem almost to be programmed individually, each to move and direct its axon (and dendrites) along an elaborately predetermined course". If even a single of the billions of neurons knows where to place itself during and after embryogenesis, then surely each of the 100,000 billion body-cells, coming off a single zygotic cell knows where to place itself, how and how long, given its place in a cytogalaxy where no two cells are riveted or pasted to each other and yet all stay put from conception to cremation, without denying rapid travel throughout the body to many a cell-type. Integrity is an animal's total noumenal blueprint that precedes, accompanies, and succeeds the animal body and one which knows how far to heal a wound or a gap whereby the body is restored to status quo ante as near to the original as possible. Multiple aphthous ulcers in the mouth, needing billions of cells to repair, get healed impeccably, and smoothly, leaving no rubble strewn around. Ditto for the BA, single/ multiple, small/large, of whatever origin, integrity orders reactivity and restorativity to so work that the least of the brain remains compromised after the BA has been therapeutically dealt with.

Given the long and ever-expanding list of autoimmune disorders, modern medicine knows not whether immunity is a friend or a foe! Medawar, the co-Nobelist with Burnet, sums up the perverted parlance of immunology as barefaced and silly, whereas Glemser, a US journalist, sizes up modern medicine's predicament by his generalization that the science of immunology is so advanced that one immunologist can't comprehend what another is talking about. Some principled parlance is in order.

Munis means duty/service. Im- in immunity is negation, and implies freedom from service, or from having to work. Immunity, then, means non-reactivity or absence of munity. The Burnet-Medawar symphony played the tune that the human embryo, circa fourth month of intrauterine life, eliminates from its thymus clones that could possibly react against the body's cells and elements. Thereafter, the so-called immune system knows which part of the body is self, and what is not-self. A self-marker or a suimarker is endowed to each self-element, an advantage not denied to an alien unit or cell present at the time of thymic maturation. Hence the acceptance of grafts, experimentally, or in monozygotic/dizygotic twins sharing a placenta, between individuals so involved. Immunity, in its principled, etymologic sense is aristocratic nonreactivity. Brain, as a tissue, is notorious for not reacting to deliberately inoculated organisms. Hence, the rarity 
of BA despite so much of otitis, sinusitis, septicemias and pyemias. BA illustrates immunity by choosing not to occur, despite the all too common head-injuries, involving a lot of road muck getting into the brain, with bony sequestra strewn around.

The word munity is best understood by the close parallel between immorality versus morality, and immunity versus munity. Munity, allied to munition or arms, is synonymous with reactivity of the animal body against non-self elements. The arsenal comprises cells and humors (antibodies), and the system is best acronymized as CHRIST - Cellulo Humoral Reactivity Insuring Somatic Totality.

As and when the brain has to react against a microbial alien, the reactive or munitive forces set up a focus of inflammation (cerebritis or cerebellitis), followed by the creation of a barricade all around to form the so-called capsule of the abscess, that now lyzes body's leucocytes and co. to create a fluid-focus called an abscess, that expanding hydrodynamically is capable of finding its way to the exterior, a phenomenon as common amongst humans as amongst animals. But in the case of BA, the walls that it must penetrate through are the vital and fragile brain tissue itself. So BA asks the fibroblasts of the vessels in the capsule-wall to generate enormous armor of collagen- collagenization- that permeating the abscess makes BA into more of a tumor than a fluid-filed abscess, more of a pyoma than a pyocoele, and a pyoma so discreetly separated from the brain tissue around, that a pyomectomy can be carried out with ease, given the plane of cleavage that separates the BA from the rest of the brain..$^{[1]}$ It must be clear to us all that CHRIST when operative in BA exercises reasoned-reactivity, and mindful-munity, whereby the BA, indistinguishably behaving clinically and radiologically as a tumor, alerts the patient and the clinician into the triad of THEOSSLAM-AAAA, assuring thereby rapid recovery. Even a BA thinks, reasons, and reacts to assure minimal brain damage, and rapid, maximal recovery.

The reactivity attending BA has had to be honored as reasoned, for the forces of CHRIST, of reactivity, are enormous. One has just to think of severe SLE, a form of contra-auto-reactive-syndrome (hitherto called autoimmune syndrome), and relentless graft-rejection on the other to appreciate the power that CHRIST can, and does, wield. It is, to use the framed phrase of Lord Tennyson, Nature, red in tooth and claw. The reasoned nature of CHRIST is BA, and so often as elsewhere, is functioning integrity, that converts CHRIST into a vector imparting to it direction, magnitude, modes, and a switch-off. Once the alien contents of an abscess cavity or the abscess itself are out, what is left is a mass of native neuraxial and guest reactive cells, all self, and hence no longer in need of any more reactivity. So hereafter, what sets in is the restorative or restitutive power of the animal body, albeit under the all-seeing eye of integrity.
An Oxford dictionary synonymizes the Latin phrase restituto in integrum as restoration to the uninjured state or restoration of the status quo ante, meaning the previous position. Be it bone, brain, bone-marrow, or liver or lung, integrity, having for itself the original blueprint of the body, orders the restorative powers to aim at reducing the area of injury/inflammation/wound/ abscessing to its barest minimum, and then offer secure repair to almost original state. In BA, once the abscess contents/abscess itself are out, the remaining neuraxial tissue, or the walls of the BA that has been aspirated, set about working to leave behind, at the most, a fibrous cicatrix, that calls for antiepileptics for a year or two. Needless to say that integrity imparts to restorativity direction, magnitude and components and switch-off as it does to reactivity. If a liver is excised $25 \%$, restorativity goes into action to replace whatsoever liver has been lost, no more, no less. In the neuraxis, neurons lost are lost forever. Yet integrity strives to replace the gap by reactive gliosis if need be. The attempt all the time is to restore the integrity of the brain to status quo ante.

The rarity of $\mathrm{BA}$ is turning into its commonness because of modern therapies 'immunocompromising' the patients, young and old. Here you see a paradox. On the one hand, you claim suppression of immunity as responsible for the setting up of inflammation, abscess, pus, et seq. On the other hand, these events proclaiming the active manifestations of the forces of 'immunity' occur widely in the body and the brain. In reality, there is no immunosuppression, or, more correctly, reactosuppression or CHRIST-letdown. What is at stake is the compromised skin/mucosal lining, because of the indiscriminate cytotoxic nature of the so-called immunosuppressors. The result is SICKNESSStructural Integrity Compromised Kemically, a state that drives the innocent bacterial symbionts deeper into where they ordinarily never are. The body has no other recource but to request CHRIST to go into action to set up BA and what have you. Unless the body has enough reactocytes (immunocytes), how would even an abscess form! SICKness opportunizes the innocent microbes which then get condemned as opportunistic. To borrow Shakespearean felicity, "The fault, dear Brutus, is not in our microbial-friends, but the compulsion to which they are iatrogenically subject". The pancytotoxic nature of the therapeutic immunosupressors also knocks out the elements of bone-marrow thus accounting for the various hemocytopenias. One can generalize that BA is more due to SICKness, than due to suppression.

$\mathrm{BA}$ is a good teacher. We better learn a lesson or two from one of the Nature's wonders called BA.

\section{Reference}

1. Kothari M, Goel A. A cogent clarifier of the confused concept of immunity. Neurosurg Focus 2008;24:E16. 